\title{
INVESTIGACIONES
}

\section{Concepciones y prácticas en la enseñanza universitaria de la física: un estudio de casos en la formación inicial docente}

\author{
Conceptions and practices in university teaching physics: \\ a case study in initial teacher training \\ Pamela Palomera-Rojas ${ }^{a, b}$, Carolina Martínez Galaz, \\ José L. Carvajal Salamanca ${ }^{d}$ \\ ${ }^{a}$ Departamento de Física. Facultad de Ciencias Básicas. \\ Universidad Metropolitana de Ciencias de la Educación. \\ ${ }^{b}$ Facultad de Educación. Pontificia Universidad Católica de Chile. \\ pamela.palomera@umce.cl \\ ${ }^{c}$ Departamento de Biología y Química. Facultad de Ciencias Básicas. \\ Universidad Católica del Maule. cmartinezg@ucm.cl \\ ${ }^{d}$ Laboratorio de Limnología. Facultad de Ciencias. Universidad de Chile. \\ jcarvajalsalamanca@u.uchile.cl
}

\begin{abstract}
RESUMEN
En esta investigación cualitativa el objetivo principal fue indagar en las concepciones y prácticas de profesores universitarios de física que forman a futuros docentes, caracterizando su visión acerca de la ciencia, su enseñanza y el aprendizaje de la disciplina. Para esto, se consideró principalmente la observación sistemática de sus clases, profundizando los principales hallazgos mediante entrevistas a los profesores y a sus estudiantes. Los resultados evidencian coexistencia de visiones epistemológicas, dadas por visiones empiristas y constructivistas respecto de la generación del conocimiento científico y visiones constructivistas relacionadas con la enseñanza y aprendizaje de la física. Respecto de las prácticas docentes, se identificaron características tales como estar basadas en la importancia de la organización de contenidos disciplinares, construcción de significados junto con sus estudiantes y utilización de modelos matemáticos para la elaboración de explicaciones. Los estudiantes reconocen un vínculo virtuoso con los profesores universitarios, impactando positivamente en su aprendizaje.
\end{abstract}

Palabras claves: Concepciones epistemológicas, Concepciones didácticas, Prácticas docentes, Enseñanza de la Física, Formación Inicial Docente.

In this qualitative research the main goal was to investigate the conceptions and practices of university teachers of physics who train future teachers, characterizing their vision about science, its teaching and the learning of the discipline. For this, the systematic observation of their classes was mainly considered, deepening the main findings through interviews with teachers and their students. The results show the coexistence of epistemological views, given by empiricist and constructivist views with about the generation of scientific knowledge and constructivist views related to the teaching and learning of physics. With regard to the teaching practices, were identified some features such as being based on the importance of the organization of disciplinary content, construction of meanings together with their students, and the use of mathematical models for the elaboration of explanations. The students recognized a virtuous link with university teachers, which would positively impact their learning.

Keywords: Epistemological Conceptions, Teaching Conceptions, Teaching Practice, Teaching Physics, Teacher Training. 


\section{INTRODUCCIÓN}

En los últimos años, la formación universitaria ha experimentado profundos cambios, modificando la estructura, roles y funciones de la Universidad como institución formadora, impactando el trabajo de los profesores, futuros profesores y la evaluación de la calidad en estos contextos formativos (Lamarra, 2015; UNESCO, 2016; Zabalza, Cid \& Trillo, 2014), y más aún para aquellos que se desarrollan en algunos programas de formación inicial docente (González-Vallejos, 2018). El profesorado en la universidad, debido a las demandas sociales y culturales, ha tenido que enfrentar un nuevo escenario formativo, lo cual ha definido un nuevo perfil docente para la formación inicial que debe atender a los procesos de enseñanza - aprendizaje disciplinares, didácticos, pedagógicos y prácticos de manera integrada (Ávalos, 2014), tensionando muchas veces sus propios roles e identidades (Radovic, Peñafiel, San Martín, Bustos \& Martínez; 2018) y por ende, el ejercicio de su profesión (Espejo \& González, 2014).

Actualmente, se reconoce que el profesorado en la educación superior debe ejercer su profesión en escenarios desafiantes tales como, aulas sobrepobladas, estudiantes diversos, curriculum con escasa articulación disciplinar y didáctica, y una cultura universitaria centrada en la valoración del desarrollo de la investigación por sobre la docencia (Guzmán, 2018). También existe consenso entre la comunidad de investigadores y académicos, acerca de la escasa investigación en Latinoamérica respecto al formador de futuros profesores, reconociendo algunos desafíos en la profundización de sus trayectorias profesionales y acerca de la docencia que realizan con sus estudiantes de pedagogía en el aula (GonzálezVallejos, 2018; Martínez \& González, 2014; Vaillant \& Marcelo, 2015).

Es así, que el ámbito de los procesos de enseñanza - aprendizaje de la ciencia en la universidad, no ha quedado exento del análisis, asumiendo que la tarea demandada al profesorado exige algunos cambios e innovaciones en las prácticas docentes universitarias (Didriksson, 2016); en concreto asumir modelos formativos que tiendan a la construcción de nuevas competencias, por parte del estudiantado y que permitan aprender de manera permanente (Benítez \& Mora, 2010; Dima, Girelli \& Reynoso Savio, 2012; Valerio \& Rodríguez, 2017). Esta necesidad de cambio en la práctica, conlleva de manera implícita que los profesores universitarios elaboren y resignifiquen sus conceptualizaciones acerca de la enseñanza y el aprendizaje de la ciencia (Fuentes, Esteban \& González, 2016; Porlán \& Martín del Pozo, 2002), instándoles a establecer nuevas concepciones y acciones de enseñanza y aprendizaje en el aula (Martínez \& Medina, 2017), planteando al mismo tiempo el desafío de recoger aún más información acerca de sus acciones formativas, práctica docente y su potencial impacto en la formación de futuros profesores de ciencia.

\section{APRENDER Y ENSEÑAR FÍSICA: CONCEPCIONES Y PRÁCTICAS}

El profesorado durante su vida profesional construye diversas concepciones para explicar el entorno que les rodea, así como también, interpreta situaciones didáctico-pedagógicas relacionadas con su propia práctica docente (Taylor \& Booth, 2015;Valerio \& Rodríguez, 2017). Los modelos seleccionados y utilizados por el profesorado, para la enseñanza y aprendizaje de la ciencia, responden a algunas de sus creencias y concepciones, desencadenando un conjunto de acciones en sus prácticas, que pueden o no ajustarse al 
contexto y a las necesidades de sus estudiantes (Sahin, Deniz \& Topçu, 2016; Zabalza et al., 2014). En este marco, se entiende a las concepciones como un conjunto de visiones y/o teorías que las personas elaboran para comprender el mundo y que responden a una estructura global de interpretaciones que abarca las creencias, los significados y las preferencias, las que influyen a su vez, sobre las acciones y decisiones a seguir (Bahçivan, 2016; García, Sanz y Vilanova, 2016). Junto con ello, se asume que las prácticas docentes derivadas de las concepciones serían el conjunto de acciones realizadas por el profesorado para propiciar el aprendizaje de los estudiantes, bajo un enfoque de práctica enriquecedora (Bain, 2006). Esto es, formar a los estudiantes desde una perspectiva integral que desarrolla procesos de alto valor educativo (Fuentes, Esteban \& González, 2016; Contreras, 2017).

También, es importante considerar que la práctica docente, integra la práctica de enseñanza, la práctica del trabajo colaborativo junto con otros profesores y el intercambio de experiencias en el contexto educativo con diferentes actores (Saroyan \& Trigwell, 2015), que a su vez pueden explicitarse desde un contexto declarativo (dadas por el propio profesorado) o bien, reconocerse producto de la observación sistemática de estos procesos (Jarauta, Medina \& Mentado, 2016).

En este contexto, es conocido que la enseñanza de la ciencia y de la física en particular, se puede analizar desde una concepción contemporánea, asociada a modelos constructivistas, así como desde una mirada tradicional, asociada a los modelos expositivos. Cuando se observa desde los modelos constructivistas, los profesores podrían tener concepciones en las cuales consideran a los estudiantes como responsables de su propio aprendizaje (Alt, 2018; Benítez \& Mora, 2010) y a la docencia, como un proceso de transformación del conocimiento; que utiliza una metodología que tiene en cuenta el qué, el cómo y para qué aprenden los estudiantes, incorporando las experiencias de estos, para relacionarlas con situaciones de la vida cotidiana. Bajo este enfoque, son los profesores quienes guían a sus estudiantes en su aprendizaje, siendo un mediador en el aula, y a su vez influenciado por sus propias creencias y cultura (Feixas, 2010; Mas-Torelló \& Olmos-Rueda, 2016), asumiendo incluso una perspectiva evolutiva y tentativa del conocimiento científico (Lederman, 2019).

Por otra parte, desde los modelos tradicionales de enseñanza, el profesorado podría concebir que ellos son los principales actores, quienes organizan y transforman el conocimiento y quienes dejan escasos espacios de autonomía a los estudiantes (Guirado, 2016). La visión más frecuente, desde este enfoque, acerca de la disciplina, es que existe un cuerpo teórico y conceptual científico moldeado y elaborado por grandes pensadores, con énfasis en modelos empíricos, los cuales deben ser transmitidos a los estudiantes (Gargallo, Fernández \& Jiménez, 2007), relegando la enseñanza y el papel del profesor a la transmisión de ese conocimiento. La interacción entre el profesorado y sus alumnos adquiere un enfoque lineal, expresado en prácticas de enseñanza con escasa retroalimentación para la comprensión de la ciencia por parte del estudiantado (Alt, 2018).

Respecto de las concepciones didácticas acerca del aprendizaje y enseñanza de la ciencia, se reconoce que los modelos han ido cambiando a través del tiempo, desde un modelo tradicional que pone énfasis en el cambio de las conductas observables producto de las experiencias (Alt, 2018), hacia modelos de aprendizaje activo centrados en el estudiante, mediante la resolución de problemas, prácticas experimentales, desarrollo de la argumentación científica, entre otras acciones (Bathgate, Crowell, Schunn, Cannady \& Dorph, 2015; Lorenzo, Farré \& Rossi, 2018). En este escenario, en el área de la enseñanza de la física se valoran prácticas orientadas al desarrollo del pensamiento crítico del 
estudiantado, el trabajo práctico-experimental, la relevancia del uso de modelos matemáticos y la capacidad de abstracción, para la comprensión de los fenómenos físicos, además de la incorporación de las tecnologías y ambientes virtuales en la enseñanza (Hernández-Silva \& Tecpan, 2017; Daineko, Dmitriyev \& Ipalakova, 2016), tal como se resume en la tabla 1.

Tabla 1: Prácticas en la enseñanza de la física

\begin{tabular}{|c|c|}
\hline Acciones & Descripción \\
\hline Resolución de problemas & $\begin{array}{l}\text { Es importante tener en cuenta que para la resolución de problemas se } \\
\text { encuentran dos perspectivas: (a) problemas cualitativos que pueden } \\
\text { ser resueltos a partir de los propios conocimientos, por medio de } \\
\text { razonamientos teóricos y sin necesidad de realizar cálculos } \\
\text { numéricos o evidencias experimentales. Este tipo de problemas } \\
\text { permite que el estudiante reflexione acerca de sus conocimientos } \\
\text { personales y sobre sus propias teorías; y (b) problemas cuantitativos } \\
\text { donde se trabaja con datos numéricos para alcanzar una solución, lo } \\
\text { que no implica que el resultado deba ser cuantitativo. Las estrategias } \\
\text { se centran en la utilización de fórmulas, cálculos numéricos o } \\
\text { comparación de datos y sirven para que el estudiante desarrolle } \\
\text { técnicas y algoritmos que le permitan abordar problemas más } \\
\text { complejos y comprender las leyes de la naturaleza (Pozo \& Gómez } \\
\text { Crespo, 1998; Vizcaino \& Terrazzan, 2015). }\end{array}$ \\
\hline $\begin{array}{l}\text { Pensamiento crítico y la } \\
\text { argumentación científica }\end{array}$ & $\begin{array}{l}\text { Para que el estudiante desarrolle un pensamiento crítico es preciso } \\
\text { que desarrolle habilidades tales como: interpretar información, } \\
\text { analizar evidencia, evaluar, inferir, explicar y autorregular su propio } \\
\text { aprendizaje. En tal contexto, la argumentación científica tiene un rol } \\
\text { importante ya que, a través de esta estrategia, el estudiantado genera } \\
\text { y evalúa evidencias que respaldan enunciados, que pueden contribuir } \\
\text { al trabajo con temáticas científicas, socio-científicas o socio- } \\
\text { económicas-ambientales (Bathgate et al; 2015). En este contexto, se } \\
\text { valora desde la enseñanza de la física el desarrollo de la habilidad } \\
\text { argumentativa, expresada en intervenciones discursivas que } \\
\text { promueven la función metacognitiva del pensamiento por parte de los } \\
\text { estudiantes, generando cambios en sus recursos explicativos (Ramos, } \\
\text { Stipcich, Domínguez \& Mosquera; 2017). }\end{array}$ \\
\hline Trabajos prácticos & $\begin{array}{l}\text { Mediante los trabajos prácticos se propone que los estudiantes } \\
\text { guiados por el profesor, descubran los hechos, conceptos y leyes a } \\
\text { través de los procesos científicos. El foco es involucrar a los } \\
\text { estudiantes en una investigación que sea parte de su contexto e interés } \\
\text { y que, mediante su desarrollo, se potencien habilidades propias del } \\
\text { quehacer científico. En tal sentido, destacan el desarrollo de } \\
\text { actividades orientadas a que los estudiantes elaboren preguntas } \\
\text { científicamente, dando paso a la recogida de pruebas y evidencias que } \\
\text { permitan dar explicaciones y al mismo tiempo, evaluar, comunicar y } \\
\text { justificar las explicaciones propuestas. (Bevins \& Price, 2016). }\end{array}$ \\
\hline
\end{tabular}




\begin{tabular}{|l|l|}
\hline $\begin{array}{l}\text { Modelos matemáticos y } \\
\text { abstracción }\end{array}$ & $\begin{array}{l}\text { Un modelo es una representación abstracta de un conjunto de } \\
\text { interacciones que delimitan al objeto de estudio. En ciencias y } \\
\text { particularmente en física la utilización de modelos matemáticos es la } \\
\text { forma en que se realiza una representación de la relación entre los } \\
\text { supuestos teóricos y la actividad experimental. La matemática en tal } \\
\text { contexto, y el uso de modelos se constituye como una herramienta de } \\
\text { apoyo a la disciplina formativa, en donde los conocimientos son } \\
\text { integrados, destacando la funcionalidad del conocimiento matemático } \\
\text { según sus usos (Camarena, 2008). }\end{array}$ \\
\hline $\begin{array}{l}\text { Uso de TIC y de entornos } \\
\text { virtuales para el } \\
\text { aprendizaje }\end{array}$ & $\begin{array}{l}\text { Ambientes que incorporan las TIC en la enseñanza y la utilización de } \\
\text { ambientes virtuales, potencian el pensamiento crítico en los } \\
\text { estudiantes, así como también el trabajo colaborativo; sobre todo en el } \\
\text { marco de modelos de aula invertida. Así, al usar plataformas virtuales, } \\
\text { bajo este enfoque, se facilita el acceso a los contenidos por parte de } \\
\text { los estudiantes durante todo el proceso de aprendizaje, propiciando } \\
\text { que construyan su propio conocimiento en espacios autónomos (fuera } \\
\text { del aula) y al mismo tiempo, junto a sus pares y profesorado (en el } \\
\text { aula). La principal contribución que recoge este enfoque, es favorecer } \\
\text { la autorregulación del aprendizaje de los estudiantes (Hernández- } \\
\text { Silva \& Tecpan, 2017; Mingorance, Trujillo, Cáceres \& Torres, 2017) }\end{array}$ \\
\hline
\end{tabular}

Fuente: Elaboración propia.

En lo que respecta a investigaciones en el área, desde hace varias décadas se reconoce la relevancia de indagar en las concepciones y creencias del profesorado acerca de cómo se enseña y aprende la ciencia y la posible relación entre las concepciones y prácticas (Bahçivan, 2016; Porlán \& Martín del Pozo, 2002; Siso \& Cuellar, 2017). En este escenario, tal como lo explica Feixas, (2010), los estudios acerca de las concepciones en enseñanza superior, y aquellos que se basan en el estudio de las concepciones a partir de la observación de la práctica docente, son menos conocidos dado que, por lo general, las concepciones se recogen desde el análisis discursivo del propio profesorado (Prieto, 2007).

Desde hace algún tiempo, varias investigaciones identifican la predominancia de visiones empiristas del conocimiento científico con la presencia de incoherencia discursiva y práctica entre las concepciones acerca del aprendizaje y las acciones de enseñanza en el aula (Porlán \& Rivero, 1998). Otros estudios, evidencian algunas concepciones del profesorado, que incluyen la valoración de la ciencia focalizada en la utilización de procedimientos rígidos y sin posibilidad de flexibilidad, tomando como eje, una secuencia de pasos dados por el método científico (Zelaya \& Campanario, 2001). En el ámbito de las concepciones epistemológicas y didácticas de profesores universitarios de ciencia, algunos estudios identifican una relación entre la visión empirista de la ciencia y la visión del aprendizaje, basado en el modelo de transmisión-recepción (Martínez \& González, 2014).

También se reconoce la existencia de mixtura y cierta heterogeneidad entre las concepciones del profesorado, respecto de algunos elementos asociados a la epistemología de la ciencia, así como, algunos relacionados con las concepciones acerca de su enseñanza y aprendizaje (Vergara \& Cofré, 2014). Es decir, los profesores pueden tener una visión constructivista del aprendizaje de la ciencia, pero al momento de enseñar, sus prácticas se 
rigen por modelos tradicionales y expositivos en el aula (Porlán \& Martín del Pozo, 2002). El estudio de Basto (2011), realizado con profesores universitarios identificó esta coexistencia de visiones a nivel de concepciones y prácticas, y en general se describieron a sí mismos como profesores activos y con enfoque constructivista, pero al observar sus clases, la investigadora evidenció que estas eran tradicionales. Este resultado, es reforzado por la contribución de Hativa, Barak \& Simhi (2001), quienes investigaron en profundidad a cuatro profesores universitarios reconocidos como ejemplares, concluyendo que, si bien existe cierta relación entre las concepciones y prácticas, éstas no son absolutas. Ravanal, Camacho, Escobar \& Jara (2014), sugieren que, en el contexto universitario, las creencias del profesorado se centran en enseñar para la adquisición del aprendizaje, utilizando modelos que buscan la apropiación de saberes, pero no la comprensión en profundidad por parte de los estudiantes.

El estudio realizado hace más de veinte años por Mellado (1996), ya reconocía la importancia de que los formadores de futuros profesores de ciencia en los contextos universitarios, generen acciones de enseñanza en coherencia con modelos didácticos que promuevan el aprendizaje profundo y significativo por parte de los estudiantes, destacando que los futuros profesores deben tomar como referencia dichos modelos, una vez inician su trabajo en la escuela. Así mismo, otras investigaciones dan cuenta de una posible asociación entre las concepciones y los años de experiencia profesional de los profesores, las cuales serían menos tradicionales en estudiantes de pedagogía o en aquellos profesores con menores años de docencia en el aula (Porlán \& Rivero, 1998).

De esta forma se plantea el desafío de profundizar en las concepciones y prácticas docentes de profesores de ciencia, y en particular en el ámbito de la formación inicial docente, reconociendo que los modelos de enseñanza adoptados por el profesorado, están estrechamente relacionados con sus concepciones acerca de la ciencia y de los procesos de enseñanza y aprendizaje de la ciencia, además de sus propias experiencias construidas desde su formación inicial y durante su desarrollo profesional (García-Carmona, Criado \& Cruz-Guzmán, 2018; Martínez \& Medina, 2017; Ravanal et al 2014; Taylor \& Booth, 2015; Zabalza et al, 2014 ).

Los antecedentes expuestos, conducen esta investigación que tiene como objetivo indagar en las concepciones y prácticas de profesores universitarios de física que forman a futuros docentes, caracterizando su visión acerca de la ciencia, su enseñanza y el aprendizaje de la disciplina. Adicionalmente, esta investigación permitiría contribuir al conocimiento del formador de profesores de física en Chile, mediante la observación de sus prácticas de enseñanza en el aula de ciencias en el contexto universitario. A partir de ello, las preguntas que orientan este estudio son: ¿cuáles son las concepciones que tienen los profesores universitarios de física, que forman a futuros docentes, acerca de la ciencia y de su enseñanza y aprendizaje? y ¿cuáles son los elementos de la enseñanza de física, que están presentes en la práctica docente del profesorado universitario en la formación inicial de profesores?

\section{METODOLOGÍA}

Esta investigación se desarrolló bajo el contexto de una tesis de magíster en didáctica de las ciencias experimentales, durante los años 2015-2016, desde un enfoque de investigación cualitativo, por medio de un estudio de casos (Stake 2007; Yin, 2018). El principal objetivo del estudio fue indagar en profundidad sobre las concepciones acerca de la ciencia y su 
enseñanza, y las prácticas docentes del profesorado universitario de física. Para esto, se realizó observación de clases de los profesores participantes en el estudio, además de entrevistas semiestructuradas a los mismos profesores universitarios y entrevistas escritas a sus estudiantes.

\subsection{PARTICIPANTES}

La selección de los participantes del estudio fue de manera intencionada (Flick, 2015) y se orientó por los siguientes atributos: (1) ser profesores universitarios pertenecientes a universidades del Consejo de Rectores de Chile (CRUCH), públicas y/o privadas, y cuyas carreras de pedagogías en las que imparten docencia estén acreditadas por la Comisión Nacional de Acreditación de Chile (CNA); (2) localizados en la región Metropolitana; (3) profesores universitarios que contaban con formación disciplinar en el área de física y también en el área de la pedagogía y/o didáctica de la física; (4) que tuviesen diferentes años de experiencia profesional en docencia universitaria; (5) que impartieran docencia en el área de física en la formación inicial de profesores de especialidad, así como también en el área de educación básica; y (6) que accedieran de forma voluntaria a participar del estudio y al registro sistemático de sus prácticas en el aula, mediante la observación de clases.

De esta forma se accedió, a tres profesores universitarios chilenos del área de física, con diferentes perfiles:

1. El profesor $1^{1}(\mathrm{P} 1)$ es licenciado en física y cuenta con estudios de postgrado (doctorado) en el área de física aplicada. Es profesor asistente y un activo investigador en física de materiales. Cuenta con productividad científica en el área. Tiene formación complementaria relacionada con la enseñanza de la física, imparte docencia en una universidad privada sin fines de lucro del CRUCH desde hace 8 años, realizando clases en las carreras de pedagogía en física y pedagogía en educación general básica. Participa de mesas de trabajo a nivel nacional relacionadas con la formación de profesores de física y también cuenta con experiencia en proyectos relacionados con la innovación docente.

2. El profesor $2(\mathrm{P} 2)$ es profesor de física y ciencias naturales y cuenta con estudios de postgrado (magíster y doctorado) en el área de la física y astrofísica. Investiga en temas relacionados a las estrellas variables y sistema solar. También, trabaja en la línea de la enseñanza de la astronomía. Cuenta con una experiencia docente universitaria de más de 20 años, impartiendo clases a futuros profesores de física en una universidad pública en la región metropolitana, de la cual es profesor titular. También se ha desempeñado en cargos de gestión y dirección académica universitaria.

3. El profesor 3 (P3) es licenciado en física y magíster en educación, del área curriculum. Durante más de 40 años se ha dedicado a la enseñanza de la física en la formación de profesores de la especialidad, vinculándose al área de la didáctica de la física en una universidad pública de la región metropolitana. Cuenta con experiencia en gestión universitaria, ya que se ha desempeñado como directivo en las carreras de pedagogía en física. Es profesor titular de la institución a la que pertenece.

Para resguardar la identidad de los participantes de este estudio es que se señalan los participantes como profesor 1 (P1), profesor 2 (P2) y profesor 3 (P3). 


\subsection{RECOLECCIÓN DE INFORMACIÓN}

El trabajo de campo se realizó principalmente durante el segundo semestre académico del 2015 y se extendió hasta los primeros meses del año 2016. Antes de iniciar el trabajo de campo se sostuvieron reuniones con los profesores universitarios participantes y también con sus estudiantes; con la finalidad de explicar los alcances del estudio y contar con el resguardo ético de la investigación, mediante la firma de consentimientos informados.

Una vez concluido este proceso, se inició la recogida de información (ver tabla 2 y tabla 3) mediante las siguientes técnicas:

a) Observación no participante de clases, proceso de observación abierta y sistemática llevado a cabo de manera intensiva hasta alcanzar el punto de saturación de los datos a los tres meses. Las observaciones de clases fueron registradas en videos y también se elaboraron notas de campo. El total de horas de observación fue de 69 horas entre los tres profesores universitarios.

b) Entrevistas semiestructuradas al profesorado, utilizando una modalidad de conversación guiada. En dichas entrevistas se recogió información sobre las concepciones epistemológicas y las prácticas docentes en las clases de física, utilizando para ello un guión que fue previamente validado mediante juicio de expertos. Se realizaron 4 entrevistas totales.

c) Entrevistas escritas a 50 estudiantes, las que se realizaron posteriormente al proceso de observación de clases. El objetivo principal fue triangular la información relacionada con las prácticas docentes de los profesores universitarios. El guión utilizado fue previamente validado mediante juicio de expertos.

Tabla 2. Información recolectada en el estudio según técnicas e informantes

\begin{tabular}{|l|c|l|}
\hline $\begin{array}{c}\text { Técnica de recogida } \\
\text { de información }\end{array}$ & $\begin{array}{c}\text { Informantes } \\
\text { claves }\end{array}$ & \multicolumn{1}{|c|}{ Información recolectada } \\
\hline $\begin{array}{l}\text { Observación no } \\
\text { participante de clases }\end{array}$ & P1 & $\begin{array}{l}\text { Concepciones acerca de la ciencia, su enseñanza y } \\
\text { aprendizaje para el área de la física. } \\
\text { Prácticas de enseñanza de la física }\end{array}$ \\
\hline Entrevistas & P3 & Discursos argumentativos acerca de sus concepciones y \\
semi-estructuradas & P2 & prácticas de enseñanza en el aula. \\
\hline Entrevistas escritas & 50 estudiantes & Prácticas de enseñanza de la física de los profesores \\
& universitarios & universitarios \\
\hline
\end{tabular}

Fuente: Elaboración propia. 
Tabla 3. Observación no participante de clases según informantes claves

\begin{tabular}{|c|l|c|}
\hline Informante clave & \multicolumn{1}{|c|}{ Asignaturas Observadas } & Horas de registro \\
\hline P1 & Física para educación general básica & 28 horas \\
\hline $\mathbf{P 2}$ & Astronomía y gravitación & 21 horas \\
\hline $\mathbf{P 3}$ & $\begin{array}{l}\text { Taller de desarrollo del pensamiento científico } \\
\text { Mecánica }\end{array}$ & 20 horas \\
\hline
\end{tabular}

Fuente: Elaboración propia.

La información fue analizada principalmente mediante análisis de contenido, atendiendo a la segmentación inicial del cuerpo de datos (codificación y categorización de unidades de significado relevantes), para posteriormente seguir el método de las comparaciones constantes propuesto por Glaser \& Strauss (1967), e integrar analíticamente los patrones concurrentes y conceptualización teórica (Gibbs, 2012). Para la gestión de datos, se trabajó con el software Atlas-ti.

Al final del proceso de análisis de los datos cualitativos provenientes de las observaciones y entrevistas, se obtuvieron 63 categorías de análisis y 1949 unidades de significado, lo que da cuenta del volumen de datos analizados. Posteriormente, las categorías generadas de manera inductiva se reorganizaron en ocho metacategorías, dada la identificación de núcleos temáticos relevantes, de las cuales y según el contexto del presente artículo, se enfatizan los resultados relacionados con 4 grandes metacategorías (Tabla 4) que son: (a) concepciones epistemológicas (Tabla 5), (b) concepciones didácticas (Tabla 6), (c) acciones para la enseñanza (Tabla 6) y (d) vínculo educativo con los estudiantes (Tabla 7).

Tabla 4: Definiciones de metacategorías

\begin{tabular}{|l|l|}
\hline Metacategorías & \multicolumn{1}{c|}{ Definición } \\
\hline $\begin{array}{l}\text { Concepciones } \\
\text { epistemológicas }\end{array}$ & $\begin{array}{l}\text { Conjunto de visiones y percepciones del profesorado acerca de la construcción } \\
\text { del conocimiento científico y de la ciencia. Estas concepciones se relacionan } \\
\text { con su formación profesional y científica. }\end{array}$ \\
\hline $\begin{array}{l}\text { Concepciones } \\
\text { didácticas }\end{array}$ & $\begin{array}{l}\text { Conjunto de visiones y percepciones del profesorado acerca de los procesos de } \\
\text { enseñanza y aprendizaje de las ciencias, particularmente de la física y de la } \\
\text { construcción del conocimiento científico. }\end{array}$ \\
\hline $\begin{array}{l}\text { Acciones para } \\
\text { la enseñanza }\end{array}$ & $\begin{array}{l}\text { Conjunto de acciones que realizan los profesores universitarios en sus clases, } \\
\text { como parte del proceso de enseñanza - aprendizaje de las ciencias y de } \\
\text { interacción constante con sus estudiantes. }\end{array}$ \\
\hline $\begin{array}{l}\text { Vínculo con el } \\
\text { estudiante }\end{array}$ & $\begin{array}{l}\text { Relaciones entre los profesores y sus estudiantes que dan cuenta de la existencia } \\
\text { de un vínculo que puede favorecer el proceso de enseñanza - aprendizaje. }\end{array}$ \\
\hline
\end{tabular}

Fuente: Elaboración propia. 
Tabla 5: Definiciones de categorías según metacategorías concepciones epistemológicas

\begin{tabular}{|l|l|l|}
\hline Metacategorías & \multicolumn{1}{|c|}{ Categoría } & \multicolumn{1}{c|}{ Definición } \\
\hline & Causalidad & $\begin{array}{l}\text { Explicaciones científicas que dan cuenta de una relación } \\
\text { entre las variables y/o fenómenos, y el efecto producido } \\
\text { sobre el mismo. }\end{array}$ \\
\cline { 2 - 3 } $\begin{array}{l}\text { Concepciones } \\
\text { epistemológicas }\end{array}$ & $\begin{array}{l}\text { Contextualización } \\
\text { histórica de la } \\
\text { ciencia }\end{array}$ & $\begin{array}{l}\text { Sitúa las leyes y/o teorías físicas en el contexto histórico en } \\
\text { el que se desarrollaron. }\end{array}$ \\
\cline { 2 - 3 } & $\begin{array}{l}\text { Razonamiento } \\
\text { inductivo }\end{array}$ & $\begin{array}{l}\text { Razonamiento lógico utilizado por el profesor universita- } \\
\text { rio, que implica que del conocimiento de hechos u observa- } \\
\text { ciones van decantando leyes y/o teorías. }\end{array}$ \\
\cline { 2 - 3 } & $\begin{array}{l}\text { Visión de ciencia- } \\
\text { constructivista }\end{array}$ & $\begin{array}{l}\text { Percepción acerca de la ciencia y el conocimiento científico, } \\
\text { se produce a partir de una construcción social y colaborativa. } \\
\text { Consideración de factores económicos, históricos, ambien- } \\
\text { tales y socioculturales al explicar los fenómenos físicos. }\end{array}$ \\
\hline
\end{tabular}

Fuente: Elaboración propia.

Tabla 6: Definiciones de categorías según metacategorías concepciones didácticas y acciones para la enseñanza

\begin{tabular}{|l|l|l|}
\hline Metacategorías & Categoría & Definición \\
\hline \multirow{4}{*}{$\begin{array}{l}\text { Concepciones } \\
\text { didácticas }\end{array}$} & $\begin{array}{l}\text { Vínculo con la } \\
\text { escuela }\end{array}$ & $\begin{array}{l}\text { Relaciones que establece el profesor universitario con la escuela } \\
\text { al contextualizar la enseñanza y el aprendizaje de la física. }\end{array}$ \\
\cline { 2 - 3 } & $\begin{array}{l}\text { Visión de la } \\
\text { enseñanza } \\
\text {-aprendizaje } \\
\text { constructivista }\end{array}$ & $\begin{array}{l}\text { Percepción acerca de que el profesor es un mediador y guía } \\
\text { en el aprendizaje de los estudiantes, desafiándoles intelec- } \\
\text { tualmente y acompañándolos en su propia construcción del } \\
\text { conocimiento científico. }\end{array}$ \\
\hline \multirow{5}{*}{$\begin{array}{l}\text { Acciones para } \\
\text { la enseñanza }\end{array}$} & $\begin{array}{l}\text { Anticipación } \\
\text { dar los estudiantes, también a los temas a tratar durante la } \\
\text { clase o en semanas posteriores. Genera expectativas de } \\
\text { explicaciones en sus estudiantes. }\end{array}$ \\
\cline { 2 - 4 } & $\begin{array}{l}\text { Argumentación } \\
\text { teórica }\end{array}$ & $\begin{array}{l}\text { Conjuntrucción } \\
\text { de significados definiciones o demostraciones que realiza el profe- } \\
\text { sor universitario para dar validez a las leyes y/o teorías físicas. }\end{array}$ \\
\cline { 2 - 3 } & $\begin{array}{l}\text { A partir de un conjunto de ideas (aportes) propuestas por los } \\
\text { estudiantes se da sentido a una explicación o concepto físico. }\end{array}$ \\
\cline { 2 - 3 } & Demostración & $\begin{array}{l}\text { Comprobación de leyes y/o teorías a partir de evidencias } \\
\text { experimentales y/o análisis matemáticos. }\end{array}$ \\
\cline { 2 - 3 } & Recapitulación & $\begin{array}{l}\text { Repaso o recordatorio de actividades y/o conceptos trabajados } \\
\text { con anterioridad o en la misma clase. }\end{array}$ \\
\cline { 2 - 3 } & $\begin{array}{l}\text { Uso de modelos } \\
\text { matemáticos }\end{array}$ & $\begin{array}{l}\text { Utilización de ecuaciones y/o herramientas matemáticas para } \\
\text { la formulación de explicaciones. }\end{array}$ \\
\hline
\end{tabular}

Fuente: Elaboración propia. 
Tabla 7: Definiciones de categorías según metacategorías vínculo educativo con los estudiantes

\begin{tabular}{|c|c|l|}
\hline Metacategorías & \multicolumn{1}{|c|}{ Categoría } & \multicolumn{1}{c|}{ Definición } \\
\hline $\begin{array}{l}\text { Vínculo educativo } \\
\text { con los estudiantes }\end{array}$ & Guiar-conducir & $\begin{array}{l}\text { El profesorado acompaña el proceso de aprendizaje de } \\
\text { sus estudiantes otorgando valor a su participación en la } \\
\text { construcción del conocimiento científico. Les incentiva } \\
\text { a tomar un rol activo y participativo en el aprendizaje. }\end{array}$ \\
\cline { 2 - 3 } & $\begin{array}{l}\text { Vínculo profesor- } \\
\text { estudiante }\end{array}$ & $\begin{array}{l}\text { Relación educativa virtuosa la que se expresa de } \\
\text { manera bidireccional entre el profesor y sus estudiantes, } \\
\text { favoreciendo el aprendizaje y la construcción del } \\
\text { conocimiento científico. }\end{array}$ \\
\hline
\end{tabular}

Fuente: Elaboración propia.

Finalmente, para facilitar la lectura de los resultados, el sistema de notación utilizado para las citas discursivas y notas de campo que ejemplifican las unidades de significado, se conforman por una referencia numérica en la cual se identifica al profesor universitario con una letra y número (P1, P2, P3), y a la fuente de obtención de información con una letra "E" para las entrevistas, "O" para la observación de clases y "EA" para entrevistas a los estudiantes.

\section{RESULTADOS}

\section{1 CONCEPCIONES EPISTEMOLÓGICAS Y DIDÁCTICAS}

Al observar las acciones de enseñanza del profesor y registrar su discurso en clases, los resultados permiten observar cómo se configuran las concepciones acerca de la ciencia y el proceso de enseñanza-aprendizaje de los profesores universitarios. Las entrevistas permitieron profundizar en las visiones manifestadas por el profesorado.

La visión acerca de la ciencia de los profesores universitarios es distinta en P1, respecto de los profesores $\mathrm{P} 2$ y $\mathrm{P} 3$, al observar sus prácticas de enseñanza en clases. Para el caso de P1, el conocimiento científico se estructura a partir de una relación lógica de conceptos, buscando siempre la causalidad en los fenómenos que se estudian (ver tabla 8). También da cuenta que es importante ir desde los conceptos básicos u observaciones hasta llegar a las teorías, relacionándolos con modelos matemáticos, dando cuenta de un tipo de razonamiento inductivo en la construcción del conocimiento científico. 
Tabla 8: Ejemplificación notas de campo-observación de clases P1

\begin{tabular}{|l|l|}
\hline \multicolumn{1}{|c|}{ Causalidad } \\
\hline OP1 & $\begin{array}{l}\text { Construye su explicación acerca de las escalas termométricas a partir de la } \\
\text { causalidad, para esto ejemplifica a través del termómetro de mercurio, indicando que } \\
\text { la causa es el calor y la consecuencia el desplazamiento de mercurio }\end{array}$ \\
\hline OP1 & $\begin{array}{l}\text { El profesor señala la causa y el efecto que se encuentra contenido en la ecuación de } \\
\text { la ley de Hooke. Define que la causa sería la fuerza aplicada sobre el resorte, } \\
\text { mientras que el efecto producido sería la elongación o compresión del resorte. }\end{array}$ \\
\hline OP1 & $\begin{array}{l}\text { Razonamiento inductivo } \\
\text { las ecuaciones y construye una explicación a partir de los resultados. }\end{array}$ \\
\hline
\end{tabular}

Fuente: Elaboración propia.

Sin embargo, cuando se consulta respecto de su visión explícita acerca de la ciencia y su construcción; P1 considera que es un proceso de construcción dinámico, sujeto al cambio e influenciado socialmente (ver tabla 9). Esta visión, no es posible identificarla en las clases analizadas.

Tabla 9: Ejemplificación citas discursivas P1

\begin{tabular}{|l|l|}
\hline \multicolumn{2}{|c|}{ Visión de ciencia constructivista } \\
\hline EP1 & $\begin{array}{l}\text { “...bueno la construcción de la ciencia es un proceso humano es un proceso dinámico, } \\
\text { es un proceso yo diría formal, en el sentido de que establece, establece cómo decirlo, } \\
\text { una cronología de conceptos que se van construyendo poco a poco para formar el } \\
\text { gran edificio del conocimiento...” }\end{array}$ \\
\hline
\end{tabular}

Fuente: Elaboración propia

En el caso de P2 y P3, sus visiones reconocen que el contexto socio-histórico influye en el avance científico (ver tabla 10), explicitando la existencia de un vínculo entre la ciencia y el contexto socio-histórico en que esta se desarrolla, lo cual es expresado a sus estudiantes en las clases analizadas. 
Tabla 10: Ejemplificación notas de campo-observación de clases y entrevistas estudiantes P2 y P3

\begin{tabular}{|l|l|}
\hline \multicolumn{3}{|c|}{ Contextualización histórica de la Ciencia } \\
\hline OP2 & $\begin{array}{l}\text { Comienza la clase comentando que ya tienen todo el fundamento teórico detrás, } \\
\text { realiza un resumen histórico de las distintas miradas de la astronomía, donde } \\
\text { establece un nexo con la divinidad y los distintos contextos religiosos. } \\
\text { (Todo este contexto histórico es para hablar de la gravedad) }\end{array}$ \\
\hline EAP2 & $\begin{array}{l}\text { “.. lo que me llama la atención es que siempre busca algún dato, acontecimiento } \\
\text { cientíico o algún premio nobel para explicar los contenidos del ramo...” }\end{array}$ \\
\hline OP3 & $\begin{array}{l}\text { “..si no hubiese existido Einstein, ihubiésemos llegado a la relatividad? Sí, } \\
\text { hubiésemos llegado a la relatividad; estaban las condiciones, faltaba que alguien } \\
\text { juntara esos conocimientos sueltos, se trata entonces de un tipo de conocimiento que } \\
\text { está siendo construido, además ha sido construido por personas de distintos siglos, } \\
\text { de distintas culturas, de distintosidiomas, de distintos credos, o sea es espectacular...” }\end{array}$ \\
\hline
\end{tabular}

Fuente: Elaboración propia.

En lo que respecta a las concepciones didácticas, los discursos de P1, P2 y P3, dan cuenta de una visión constructivista centrada en que el profesor es un mediador en el aprendizaje de los estudiantes (ver tabla 11).

Tabla 11: Ejemplificación citas discursivas P1, P2 y P3

\begin{tabular}{|l|l|}
\hline \multicolumn{3}{|c|}{ Visión de la enseñanza-aprendizaje constructivista } \\
\hline EP1 & $\begin{array}{l}\text { "lo que trato de hacer es simplemente plantearles las expresiones matemáticas y } \\
\text { tratar de que las entiendan desde el punto de vista de la física o sea que no sea una } \\
\text { fórmula, sino que realmente vean cuáles son las relaciones entre los conceptos...que } \\
\text { los construyan" }\end{array}$ \\
\hline OP2 & $\begin{array}{l}\text { El profesor luego de dar algunas recomendaciones acerca de cómo tratar ciertos } \\
\text { contenidos cuando esté en el aula con sus estudiantes, señala que “...no se puede } \\
\text { dejar al estudiante que descubra solo, porque quedará hablando solo de lo que } \\
\text { conoce y no se dará el salto a nuevas ideas. Es necesario el acompañamiento para } \\
\text { que reciba la teoría detrás solo de esa manera, con un sistema mixto dará el salto.... }\end{array}$ \\
\hline EP3 & $\begin{array}{l}\text { “..la ciencia es un recurso pedagógico que nos permite lograr diferentes objetivos } \\
\text { educacionales, o sea yo, utilizo la ciencia, yo no soy científico, sino que sé algo de } \\
\text { ciencia...acompaño a mis estudiantes en su construcción” }\end{array}$ \\
\hline
\end{tabular}

Fuente: Elaboración propia.

En el caso de P2 y P3, aparece en las clases analizadas, un fuerte vínculo a la escuela (ver tabla 12), expresado en ejemplificaciones y situaciones que conectan el aprendizaje y enseñanza de la física en la formación que imparten a los futuros profesores. 
Tabla 12: Ejemplificación notas de campo-observación de clases P2 y P3

\begin{tabular}{|l|l|}
\hline \multicolumn{2}{|c|}{ Vínculo con la Escuela } \\
\hline OP2 & $\begin{array}{l}\text { El profesor señala a sus estudiantes que cuando realicen sus clases deben dar énfasis } \\
\text { en las leyes y teorías. Realiza recomendaciones de elementos que deben considerar a } \\
\text { la hora de hacer clases, acerca del contenido plantea que Kepler da una regularidad } \\
\text { yordenamiento al sistema solar, por tanto, se debe transmitir ese orden. }\end{array}$ \\
\hline OP3 & $\begin{array}{l}\text { El aprendizaje riguroso de las leyes físicas es válido, pero sólo para una parte de la } \\
\text { población, no para el común, ya que no todos se dedicarán a la ingeniería o a la } \\
\text { ciencia, y eso es importante tenerlo claro en la escuela. }\end{array}$ \\
\hline
\end{tabular}

Fuente: Elaboración propia.

\subsection{ACCIONES PARA LA ENSEÑANZA}

En el discurso expositivo en las clases analizadas, identificamos acciones que dan cuenta de las prácticas docentes. El participante P1, explicita la intencionalidad de organizar los contenidos en relación a dos acciones principales: la anticipación y la recapitulación de contenidos. El uso de la anticipación, este profesor lo aborda como una estrategia para despertar la curiosidad de los estudiantes, generando expectativas en las explicaciones. La acción de recapitular, por otra parte, corresponde a una recuperación de parte del contenido o experiencias vividas en la misma sesión o de clases anteriores, transformándose en un punto de transición necesario para la comprensión de los nuevos conceptos (ver tabla 13).

Tabla 13: Ejemplificación notas de campo-observación de clases y citas discursivas P1

\begin{tabular}{|l|l|}
\hline \multicolumn{3}{|c|}{ Anticipación } \\
\hline OP1 & $\begin{array}{l}\text { Luego de mostrar un estudio de aislación realizado en construcción, adelanta que el } \\
\text { concepto que trabajarán es dilatación térmica, para eso muestra la imagen de una } \\
\text { cafetera, realizando una descripción de la imagen y del sistema físico que implica. }\end{array}$ \\
\hline EP1 & $\begin{array}{l}\text { “...yo creo que eso que yo hago sobre todo en las preguntas, o sea yo les pregunto } \\
\text { para que por lo menos tengan la inquietud de la pregunta” }\end{array}$ \\
\hline EP1 & $\begin{array}{l}\text { Recapitulación } \\
\text { “... digamos resumo las cosas más importantes de la clase anterior, pero dentro de } \\
\text { previo profundizan algunos conceptos para que tengan una distinción, para que } \\
\text { tengan una concepción más profunda de las aplicaciones por ejemplo...” }\end{array}$ \\
\hline
\end{tabular}

Fuente: Elaboración propia. 
En el caso de P2 y P3 se obtuvo como resultado que sus acciones de enseñanza consideran la utilización de argumentaciones teóricas al explicar conceptos físicos, así como la importancia atribuida a la demostración y uso de modelos matemáticos para generar estructuras de comprensión de leyes y teorías físicas (ver tabla 14). Estas acciones por lo general se presentan de manera integrada e interrelacionadas en el aula.

Tabla 14: Ejemplificación notas de campo-observación de clases P2 y P3

\begin{tabular}{|c|c|}
\hline \multicolumn{2}{|r|}{ Argumentación teórica/ Uso de modelos matemáticos } \\
\hline OP2 & $\begin{array}{l}\text { Explicando la conservación de la energía, el profesor plantea que existe una } \\
\text { correspondencia entre las leyes físicas, ya que se cumplen independiente del contexto. } \\
\text { El profesor escribe en la pizarra una igualdad de dos ecuaciones que aparentemente } \\
\text { son muy distintas entre sí. Luego de la explicación establece una relación entre } \\
\text { ambas y corrobora la correspondencia de las leyes físicas. }\end{array}$ \\
\hline \multicolumn{2}{|r|}{ Demostración/Uso de modelos matemáticos } \\
\hline OP3 & $\begin{array}{l}\text { El profesor para dar respuesta a uno de los problemas de la guía de taller } n^{\circ} 3 \text {, } \\
\text { realiza un vínculo entre los modelos matemáticos y lo que sucede en la realidad } \\
\text { cuando un cuerpo se coloca en movimiento, escribiendo una deducción de un } \\
\text { conjunto de expresiones algebraicas. }\end{array}$ \\
\hline
\end{tabular}

Fuente: Elaboración propia.

Se obtuvo que existe otra forma de construir explicaciones por parte de P2 y P3, esto lo realizan explícitamente a partir de las inquietudes o ideas de los estudiantes, promoviendo su participación en la construcción de significados (ver tabla 15).

Tabla 15: Ejemplificación notas de campo-observación de clases P2 y P3

\begin{tabular}{|l|l|}
\hline \multicolumn{1}{|c|}{ Construcción de significados } \\
\hline $\mathbf{O P 2}$ & $\begin{array}{l}\text { El profesor se detiene a responder las preguntas que surgen de los estudiantes, al } \\
\text { explicar los distintos casos de energía positiva o negativa realiza esquemas (dibujos) } \\
\text { en la pizarra, con sus explicaciones trata de que los estudiantes se sitúen en el caso } \\
\text { que está describiendo, mediante un ejemplo, “.... ¿qué pasaría con nosotros si nos } \\
\text { moviéramos a cierta velocidad con "x" energía?” }\end{array}$ \\
\hline OP3 & $\begin{array}{l}\text { Realiza nuevas preguntas de la interpretación del enunciado y de las condiciones } \\
\text { iniciales que se presentan, les dice a sus estudiantes: “...la ciencia es democrática...”, } \\
\text { para que se atrevan a comentar, luego él retoma los comentarios que realizan los } \\
\text { estudiantes y los integra a la traducción del enunciado }\end{array}$ \\
\hline
\end{tabular}

Fuente: Elaboración propia. 


\subsection{VÍNCULO EDUCATIVO CON LOS ESTUDIANTES}

Se obtuvo que los profesores universitarios del estudio, como parte de sus prácticas docentes, establecen relaciones y vínculos educativos con los estudiantes que ayudaban al aprendizaje de estos. En este contexto, asumimos que el discurso expositivo es en esencia interactivo, ya que depende de la participación de los estudiantes, donde se produce una influencia mutua en el marco de una acción cognitiva, por lo tanto, la calidad del discurso tiene una estrecha relación con la conexión y cooperación de los estudiantes en esta construcción. De esta forma y considerando las observaciones y visiones de los estudiantes a cargo de los profesores P1, P2 y P3, se obtiene que en forma general el discurso explicativo siempre se desarrolla en un ambiente de empatía y cordialidad por parte del profesorado. Este ambiente grato, es considerado por los estudiantes como un contexto favorable para su aprendizaje, destacando su capacidad de responder sus inquietudes y repetir las explicaciones cuantas veces sea necesario (ver tabla 16). También se reconoce un ambiente de reflexión colectiva, donde se pueden escuchar mutuamente profesor y estudiantes, para construir ideas en conjunto de manera libre y espontánea, y que da cuenta de objetivos de aprendizaje que han sido bien pensados.

Tabla 16: Ejemplificación entrevistas a estudiantes de P1 y P2 y citas discursivas de P3

\begin{tabular}{|c|c|}
\hline \multicolumn{2}{|r|}{ Vínculo Profesor-Estudiante } \\
\hline EAP1 & $\begin{array}{l}\text { "Me gusta porque es directo y claro en su forma de explicar. En el trato con sus } \\
\text { alumnos es muy cordial y cálido y da muestras claras de preocupación por el } \\
\text { aprendizaje" }\end{array}$ \\
\hline EAP2 & "Explica y vuelve a explicar las veces que sean necesarias para lograr entender" \\
\hline \multicolumn{2}{|r|}{ Guiar conducir } \\
\hline EAP1 & $\begin{array}{l}\text { "Además me ayuda el hecho que tiene un estilo muy directo de comunicarse y } \\
\text { responde a todas las inquietudes incluyendo aquellas que se escapan de la materia } \\
\text { puntual tratada" }\end{array}$ \\
\hline EAP2 & $\begin{array}{l}\text { "Constantemente nos está dando ejemplos y relaciones para poder enseñarles a } \\
\text { nuestros futuros estudiantes" }\end{array}$ \\
\hline EP3 & $\begin{array}{l}\text { “...yo parto de la base de que lo que me dice la ley cero de la pedagogía, que } \\
\text { normalmente el profesor novato trata de imitar a los profesores que lo formaron, } \\
\text { entonces toma el modelo de algún profesor y trata de ser más o menos como él. } \\
\text { Entonces uno tiene que darle no solo contenidos conceptuales sino también recursos } \\
\text { didácticos, y no como he escuchado un montón de veces, yo paso mis conceptos de } \\
\text { mecánica, unos problemas terribles y después ustedes vean con el profesor de } \\
\text { metodología cómo los aterrizan en el colegio, esa cuestión yo considero que es un } \\
\text { crimen. No, yo creo que si uno va a desarrollar la conservación del momento lineal } \\
\text { con sus estudiantes uno tiene que desarrollar cosas que sean transferibles al colegio, } \\
\text { lo cual no significa que deban ser elementales o triviales hay problemas que tú misma } \\
\text { has podido evidenciar..." }\end{array}$ \\
\hline
\end{tabular}

Fuente: Elaboración propia. 
A modo de síntesis, la tabla 17 , integra los principales resultados obtenidos para los tres profesores universitarios respecto de las concepciones y prácticas docentes.

Tabla 17: Síntesis de resultados de P1, P2 y P3

\begin{tabular}{|c|c|c|c|c|c|}
\hline Profesor & $\begin{array}{c}\text { Concepciones } \\
\text { epistemológicas }\end{array}$ & $\begin{array}{c}\text { Concepciones } \\
\text { didácticas }\end{array}$ & $\begin{array}{c}\text { Prácticas } \\
\text { docentes/ } \\
\text { Acciones de } \\
\text { enseñanza }\end{array}$ & $\begin{array}{c}\text { Prácticas } \\
\text { docentes/ Vínculo } \\
\text { educativo con los } \\
\text { estudiantes }\end{array}$ & $\begin{array}{c}\text { Años de } \\
\text { experiencia } \\
\text { profesional }\end{array}$ \\
\hline $\mathbf{P 1}$ & Empirismo & Constructivista & $\begin{array}{c}\text { Anticipación } \\
\text { Recapitulación }\end{array}$ & $\begin{array}{c}\text { Guiar-conducir } \\
\text { Empatía } \\
\text { Acompañar en el } \\
\text { aprendizaje }\end{array}$ & 8 años \\
\hline $\mathbf{P 2}$ & $\begin{array}{c}\text { Construcción } \\
\text { social del } \\
\text { conocimiento } \\
\text { científico }\end{array}$ & Constructivista & $\begin{array}{c}\text { Argumentación } \\
\text { teórica } \\
\text { matemáticos } \\
\text { Construcción de } \\
\text { significados }\end{array}$ & $\begin{array}{c}\text { Acompañar en el } \\
\text { aprendizaje }\end{array}$ & +20 años \\
\hline P3 & $\begin{array}{c}\text { Construcción } \\
\text { social del } \\
\text { conocimiento } \\
\text { científico }\end{array}$ & Constructivista & $\begin{array}{c}\text { Uso de modelos } \\
\text { matemáticos } \\
\text { Construcción de } \\
\text { significados }\end{array}$ & Muiar-conducir \\
Modelar & +40 años \\
\hline
\end{tabular}

Fuente: Elaboración propia.

\section{DISCUSIÓN}

En consideración a las concepciones epistemológicas se identifica incoherencia discursiva, dando cuenta de las mixturas ya reportadas. En el caso de P1 se evidencia una concepción cercana a una visión empirista de la ciencia al momento de enseñar física en el aula, donde se busca dar a conocer a los estudiantes un procedimiento lógico que demuestre una causalidad entre las variables; propio de una visión más tradicional (Benítez \& Mora, 2010; García, Sanz \& Vilanova, 2016) y al mismo tiempo existe una visión acerca de la ciencia como construcción social, cuando se indaga de manera explícita en sus concepciones. Tal situación se condice con lo reportado por varias investigaciones que reconocen la coexistencia de la visión acerca de la ciencia en los profesores (Bahçivan, 2016; Martínez \& González, 2014; Siso \& Cuellar, 2017).

En el caso de los profesores P2 y P3, sus visiones epistemológicas dan cuenta de visiones constructivistas, las cuales son reconocidas de manera coherente como parte de su discurso explicativo en clases, al momento de enseñar física. Estas concepciones relevan la importancia del conocimiento del contexto sociocultural en el que se desarrolla la ciencia y los favorables usos didácticos de la historia y la filosofía de la ciencia, en el área de 
física (Teixeira, Greca \& Freire, 2012), lo que está explícito en las prácticas docentes de la enseñanza de la física a sus estudiantes.

Las concepciones didácticas de los profesores universitarios son cercanas a un modelo constructivista de enseñanza y aprendizaje; destacando en dos de ellos, el establecimiento de vínculos con la escuela al momento de enseñar contenidos de física, explicitando con esto su rol en el contexto de la formación inicial, que contribuye a situar a los futuros profesores de física en contextos laborales futuros. Una posible explicación para esta acción observada en la enseñanza de la física, se puede deber a los años de experiencia profesional que tienen P2 y P3, a diferencia de P1; y dada su experiencia formativa con la que cuentan, reconocen que estos vínculos son de alto valor para los estudiantes de pedagogía.

En relación a las prácticas docentes, se evidencia que los profesores investigados privilegian acciones de enseñanza, como el uso de la matemática, por sobre las evidencias experimentales a la hora de enseñar (Zelaya \& Campanario, 2001), en lo que respecta principalmente a la resolución de problemas (Vizcaino \& Terrazzan, 2015). El fin último de los profesores universitarios no es únicamente transmitir conocimientos acabados acerca de la especialidad en física, sino también promover el interés y estimular a sus estudiantes para construir significados. Esta visión se observa principalmente en los profesores P2 y P3 quienes ponen de manifiesto la necesidad de considerar las ideas de los estudiantes como punto de partida, para generar nuevo conocimiento; sin embargo, cuando se evidencian errores conceptuales en ellos, son los profesores quienes realizan las correcciones que sean necesarias (Eder, 2017; Zelaya \& Campanario, 2001).

La realización de una constante anticipación y recapitulación de contenidos, traducción de conceptos físicos, leyes, teorías y ecuaciones matemáticas, como acciones y decisiones tomadas por los profesores universitarios, permiten simplificar dichos elementos para la comprensión por parte de sus estudiantes (Jarauta et al., 2016; Medina \& Jarauta, 2013). Todas estas acciones reflejadas en la práctica al enseñar física son subyacentes a una intención didáctica que promueve una orientación explicativa que actúa sobre las actitudes de los estudiantes, y lo que ellos conocen. Así mismo intentan generar o incrementar el interés de los estudiantes hacia los contenidos y hacia la persona que los imparte (Alt, 2018).

En relación con el vínculo educativo de los profesores con sus estudiantes, como parte de sus prácticas docentes, los resultados obtenidos en esta investigación, dan cuenta de un carácter bidireccional positivo. Los estudiantes valoran este vínculo y relación virtuosa, reconociendo en el profesorado algunas acciones que promueven un clima de confianza y que favorece el aprendizaje en el ámbito de la física (Bain, 2006; González, Martínez, Martínez, Cuevas \& Muñoz, 2009; Contreras, 2017). Esta característica es observada en los tres profesores y en sus grupos de estudiantes. Cabe destacar que, si bien estas acciones se observan en los tres profesores universitarios, se evidencian distintos estilos de enseñanza, los cuales abarcan desde enfoques más centrados en el protagonismo del profesor y otras que otorgan mayor protagonismo a sus estudiantes (Eder, 2017; Gargallo, Sánchez, Ros \& Ferreras, 2010; Zelaya \& Campanario, 2001).

Finalmente se identifica que las estrategias docentes utilizadas por los profesores universitarios a la hora de enseñar tienen un carácter relacional, ético y conversacional, producto de las experiencias pedagógicas vividas (Zabalza et al., 2014). Se infiere que mientras más experiencia docente tiene el profesorado universitario, emana de su práctica 
reflexiva una dimensión que cuestiona sus concepciones, enseñanza, vínculo educativo con sus estudiantes y su propio desarrollo profesional (Carabajo, 2008; Contreras, 2017), lo cual impactaría en las concepciones y prácticas al momento de enseñar a sus estudiantes.

\section{CONCLUSIONES}

En esta investigación, se logra obtener información importante acerca de las concepciones y prácticas de profesores universitarios de física que forman a futuros docentes. Respecto de las concepciones del profesorado, se puede concluir que son parte de una dimensión personal, afectiva, ligada al contexto en el que se desarrollan y esto también es parte de un conjunto de significados que se construyen fruto de la experiencia profesional. Tal como se ha evidenciado aquí, las concepciones influyen en las acciones de enseñanza implementadas en el aula, las cuales tienen su gestación en la formación inicial docente.

Por otra parte, en cuanto a las prácticas docentes, los profesores universitarios investigados muestran acciones de enseñanza centradas en construir significados con sus estudiantes, traducir conceptualmente los contenidos y usar diversas estrategias que facilitan la comprensión de los conceptos en el ámbito de la física. Este estudio, reconoce una relación implícita entre las concepciones y las prácticas de enseñanza de los profesores universitarios estudiados, la que depende de los años de experiencia profesional, es decir mientras más experimentados sean los profesores, es mayor la consistencia y articulación entre sus concepciones acerca de la enseñanza de la física y sus acciones implementadas en el aula.

También se logra evidenciar que la experiencia profesional docente tiene un carácter reflexivo, ya que los profesores estudiados son más conscientes de sus propias prácticas de enseñanza y capaces de argumentar sus acciones en el aula en relación a sus experiencias vividas. Esto quiere decir que, aquellos profesores con mayor experiencia profesional, tienen la capacidad de replantearse el proceso de enseñanza de la física contribuyendo al aprendizaje del estudiantado mediante prácticas que poseen un alto valor educativo.

Finalmente, los hallazgos de esta investigación, permiten proyectar la importancia de ampliar el estudio a otras disciplinas de las ciencias experimentales. Sería valioso considerar algunos de estos elementos claves acerca de las concepciones y prácticas en las escuelas formadoras de profesores, tales como los usos didácticos de perspectivas históricas y filosóficas en la construcción del conocimiento científico, la contextualización de la enseñanza de la física en la escuela, el establecimiento de vínculos educativos y pedagógicos centrados en la valoración positiva del estudiantado, así como también la instalación de prácticas docentes constructivistas que favorezcan la construcción del aprendizaje de los estudiantes. Estos elementos deben incluirse como parte de un proceso de transferencia de prácticas de alto valor educativo por parte de los formadores experimentados hacia los formadores novatos, quienes comienzan su camino en la docencia universitaria, particularmente en la formación de futuros profesores de física. El aporte de este estudio y de otras investigaciones realizadas hasta ahora, posibilita indagar aún más en el contexto universitario y en la formación inicial de profesores de física (Âvalos \& Sotomayor, 2012; Eder, 2017; Martínez \& González 2014) planteando el desafío de contribuir a la enseñanza de las ciencias y a mejoras en las prácticas docentes en la universidad (Castiblanco \& Nardi, 2012; Lorenzo et al., 2018) 


\section{REFERENCIAS BIBLIOGRÁFICAS}

Alt, D. (2018). Science teachers' conceptions of teaching and learning, ICT efficacy, ICT professional development and ICT practices enacted in their classrooms. Teaching and Teacher Education, $73,141-150$.

Ávalos, B. (2014). La formación inicial docente en Chile: Tensiones entre políticas de apoyo y control. Estudios pedagógicos (Valdivia), 40 (Especial), 11-28. https://dx.doi.org/10.4067/ S0718-07052014000200002

Ávalos, B., \& Sotomayor, C. (2012). Cómo ven su identidad los docentes chilenos. Perspectiva Educacional, 51(1).

Bahçivan, E. (2016). Investigating the relationships among PSTs' teaching beliefs: are epistemological beliefs central? Educational Studies, 42(2), 221-238.

Bain, K. (2006). Lo que hacen los mejores profesores universitarios. Valencia: PUV.

Basto, S. (2011). De las concepciones a las prácticas pedagógicas de un grupo de profesores universitarios. Magis, revista internacional de investigación en educación. 3(6), 393-412.

Bathgate, M., Crowell, A., Schunn, C., Cannady, M., \& Dorph, R. (2015). The Learning Benefits of Being Willing and Able to Engage in Scientific Argumentation. International Journal of Science Education, 37(10), 1590-1612

Benítez, Y. \& Mora, C. (2010). Enseñanza tradicional vs aprendizaje activo para alumnos de ingeniería. Revista Cubana de Física, 27(2A) 175-179.

Bevins, S., \& Price, G. (2016). Reconceptualising inquiry in science education. International Journal of Science Education, 38(1), 17-29.

Camarena, P. (2008). La matemática en el contexto de las ciencias. En Gaita, Cecilia (Ed.), III Coloquio Internacional sobre Enseñanza de las Matemáticas (pp. 83-105). Coloquio llevado a cabo en Pontificia Universidad Católica del Perú, Perú.

Carabajo, R. (2008). La metodología fenomenológica- hermenéutica de M. Van Manen en el campo de la investigación educativa. Posibilidades y primeras experiencias. Revista de Investigación Educativa, 26(2), 409-430.

Castiblanco, O., \& Nardi, R. (2012). Establishing common elements among some science education references as a resource to design a Didactics of Physics program for teachers' initial education. Lat. Am. J. Phys. Educ, 6 (Suppl I).

Contreras, J. (2017). Tensiones fructíferas: explorando el saber pedagógico en la formación del profesorado: Una mirada desde la experiencia. Ediciones Octaedro.

Daineko, Y., Dmitriyev, V., \& Ipalakova, M. (2016). Using virtual laboratories in teaching natural sciences: An example of physics courses in university. Computer Applications in Engineering Education, 25(1), 39-47.

Didriksson, A. (2016). La universidad desde su futuro. Pro-posições, 15(3), 63-73.

Dima, G., Girelli, M., \& Reynoso Savio, M. F. (2012). Aprendizaje activo de la física en alumnos de nivel secundario: Pre test de laboratorio sobre transformación de energía eléctrica en térmica. Lat. Am. J. Phys. Educ, 6(1), 143.

Eder, M. (2017). La enseñanza de la física en las clases universitarias. Las buenas prácticas. Enseñanza de las ciencias. (Extra), 2583-8.

Espejo, M. \& González Suarez, M. (2014). El desarrollo de profesores universitarios y escolares en Chile: ¿Brecha o continuidad? Perspectiva Educacional, 53(2), 3-19.

Feixas, M. (2010). Enfoques y concepciones docentes en la universidad. Revista Electrónica de Investigación y Evaluación Educativa, 16(2), 1-27.

Flick, U. (2015). El diseño de investigación cualitativa. Madrid: Ediciones Morata S.L.

Fuentes, J. L., Esteban, F., \& González, M. D. R. (2016). Sobre las dimensiones identitarias esenciales del profesorado universitario en el contexto pedagógico-tecnológico contemporáneo. Education in the Knowledge Society, 17(2), 39-53. 
García, M. B., Sanz, M. M., \& Vilanova, S. L. (2016). ¿Qué concepciones sobre el conocimiento científico tienen los docentes universitarios de ciencias? Diseño, validación y aplicación de un cuestionario de dilemas para evaluar concepciones implícitas. Revista docencia universitaria, $17(1), 17-41$.

García-Carmona, A., Criado, M., \& Cruz-Guzmán, M. (2018). Prospective primary teachers' prior experiences, conceptions, and pedagogical valuations of experimental activities in science education. International Journal of Science and Mathematics Education, 16(2), 237-253.

Gargallo, B., Fernández, A. \& Jiménez, A. (2007). Modelos docentes de los profesores universitarios. Teoría de la Educación, 19, 167-189.

Gargallo, B., Sánchez, F., Ros, C. \& Ferreras, A. (2010). Estilos docentes de los profesores universitarios. La percepción de los alumnos de los buenos profesores. Revista Iberoamericana de Educación, 51(4), 1-16.

Gibbs, G. (2012). El análisis de datos cualitativos en la investigación cualitativa. Madrid: Ediciones Morata S.L.

Glaser, B.G. y Strauss, A. (1967). The Discovery of Grounded Theory: Strategies for Qualitative Research. Chicago: Aldine

González, C., Martínez, M., Martínez, C., Cuevas, K., \& Muñoz, L. (2009). La Educación Científica como apoyo a la movilidad social: desafíos en torno al rol del profesor secundario en la implementación de la indagación científica como enfoque pedagógico. Estudios pedagógicos, 35(1), 63-78.

González-Vallejos, M. P. (2018). El estudio del formador latinoamericano: un campo de investigación 'en construcción'. Revista Internacional de Investigación en Educación, 10(21), 35-54.

Guirado, A. (2016). Los modelos didácticos de docentes de Ciencias Naturales de nivel secundario: reconstrucción a partir de sus concepciones y sus prácticas áulicas. Revista de Enseñanza de la Física, 28(2), 111-112.

Guzmán, C. (2018). Las buenas prácticas de enseñanza de los profesores de Educación Superior. REICE. Revista Iberoamericana sobre Calidad, Eficacia y Cambio en Educación, 16(2), 133149.

Hativa, N., Barak, R., \& Simhi, E. (2001). Exemplary university teachers: Knowledge and beliefs regarding effective teaching dimensions and strategies. The Journal of Higher Education, 72(6), 699-729.

Hernández-Silva, C., \& Tecpan, S. (2017). Aula invertida mediada por el uso de plataformas virtuales: un estudio de caso en la formación de profesores de física. Estudios pedagógicos, 43(3), 193-204.

Jarauta, B., Medina, J. \& Mentado, T. (2016). La transformación del saber en la enseñanza universitaria. Una aproximación desde el estudio del CDC. Revista de Investigación Educativa, 34(2), 471-485.

Lamarra, N. F. (2015). La educación superior en América Latina. Aportes para la construcción de una nueva agenda. Debate universitario, 1(1), 1-29.

Lederman, N.G. (2019) Contextualizing the Relationship Between Nature of Scientific Knowledge and Scientific Inquiry. Science \& Education 28, 249-267. https://doi.org/10.1007/s11191-01900030-8.

Lorenzo, M. G., Farré, A. S., \& Rossi, A. M. (2018). La formación del profesorado universitario de ciencias. El conocimiento didáctico y la investigación científica. Revista Eureka sobre Enseñanza y Divulgación de las Ciencias, 3603-3603.

Martínez, C. \& González, C. (2014). Concepciones del profesorado universitario acerca de la ciencia y su aprendizaje y cómo abordan la promoción de competencias científicas en la formación de futuros profesores de Biología. Enseñanza de las ciencias, 32(1), 51-81.

Martínez, C. \& Medina, J. (2017) Concepciones y prácticas del profesorado universitario de Ciencias: un estudio de casos en la enseñanza de la Biología. Enseñanza de las ciencias, (Extra), 27632768. 
Mas-Torelló, Ó., \& Olmos-Rueda, P. (2016). El profesor universitario en el Espacio Europeo de Educación Superior: la autopercepción de sus competencias docentes actuales y orientaciones para su formación pedagógica. Revista mexicana de investigación educativa, 21(69), 437-470.

Medina, J. y Jarauta, B. (2013). Análisis del conocimiento didáctico del contenido de tres profesores universitarios. Revista de educación, 360, 131.

Mellado, V. (1996). Concepciones y prácticas de aula de profesores de ciencias, en formación inicial de primaria y secundaria. Enseñanza de las ciencias, 14(3), 289-302.

Mingorance, A., Trujillo, J., Cáceres, P., Torres, C. (2017). Mejora del rendimiento académico a través de la metodología de aula invertida centrada en el aprendizaje activo del estudiante universitario de ciencias de la educación. Journal of Sport and Health Research. 9(1), 129-136.

Porlán, R \& Martín del Pozo, M. (2002). Spanish Teachers' Epistemological and Scientific Conceptions: Implications for teacher education1. European Journal of Teacher Education, 25(2-3), 151-169.

Porlán, R. \& Rivero, A. (1998). El conocimiento de los profesores. Sevilla. Diada Editorial SL.

Pozo, J., \& Gómez Crespo, J. (1998). El aprendizaje de la Física. En Pozo, J. I., \& Gómez Crespo, M. Á. Aprender y enseñar ciencia. Madrid: Morata.

Prieto, L. (2007). Autoeficacia del profesor universitario. Madrid: Narcea

Radovic, D., Peñafiel, B., San Martín, A., Bustos, S. \& Martínez, S. (2018). Enseñando matemáticas a futuros profesores en un sistema complejo: Formadores de profesores en universidades de Chile. Estudios pedagógicos (Valdivia), 44(3), 101-116. https://dx.doi.org/10.4067/S071807052018000300101

Ramos, W., Stipcich, S., Domínguez, A., \& Mosquera, C. (2017). La formación en argumentación de futuros profesores de física: revisión de estudios actuales. Revista de Enseñanza de la Física, $29,121-128$.

Ravanal, E., Camacho, J., Escobar, L., \& Jara, N. (2014). ¿Qué dicen los profesores universitarios de ciencias sobre el contenido, metodología y evaluación?: Análisis desde la acción educativa. REDU: Revista de Docencia Universitaria, 12(1), 307.

Sahin, A., Deniz, H., \& Topçu, S. (2016). Predicting Turkish Preservice Elementary Teachers' Orientations to Teaching Science with Epistemological Beliefs, Learning Conceptions, and Learning Approaches in Science. International Journal of Environmental and Science Education, 11(5), 515-534.

Saroyan, A., \& Trigwell, K. (2015). Higher education teachers' professional learning: Process and outcome. Studies in Educational Evaluation, 46, 92-101.

Siso, Z. y Cuéllar, L. (2017). Relaciones entre las concepciones de naturaleza de la ciencia y tecnología y de la enseñanza y aprendizaje de las ciencias de profesores de Química en ejercicio. Una primera aproximación al esquema conceptual del profesor. Revista de la Facultad de Ciencia y Tecnología-Tecné, Episteme y Didaxis, TED, 41, 17-36.

Stake, R. (2007). Investigación con estudio de casos. Madrid: Ediciones Morata S.L.

Taylor, L., \& Booth, S. (2015). Secondary physical science teachers' conceptions of science teaching in a context of change. International Journal of Science Education, 37(8), 1299-1320.

Teixeira, E., Greca, I., \& Freire, O. (2012). The history and philosophy of science in physics teaching: A research synthesis of didactic interventions. Science \& Education, 21(6), 771-796.

UNESCO (2016). Informe de seguimiento sobre la educación en el mundo. Creación de futuro sostenible para todos. Paris: UNESCO.

Vaillant, D. \& Marcelo, C. (2015). El A, B, C y D de la formación docente. España: Narcea Ediciones.

Valerio, G., \& Rodríguez M. (2017). Perfil del profesor universitario desde la perspectiva del estudiante. Innovación educativa, 17(74), 109-124.

Vergara, C. \& Cofré, H. (2014). Conocimiento Pedagógico del Contenido: ¿el paradigma perdido en la formación inicial y continua de profesores en Chile? Estudios pedagógicos, 40(Especial), 323-338. 
Vizcaino Arévalo, D. F., \& Terrazzan, E. A. (2015). Diferencias trascendentales entre matematización de la física y matematización para la enseñanza de la física. Tecné, Episteme Y Didaxis: TED, 38(38). https://doi.org/10.17227/01203916.3789

Yin, R. K. (2018). Case study research and applications. Los Angeles: SAGE

Zabalza, M., Cid, A. \& Trillo, A. (2014). Formación docente del profesorado universitario. El difícil tránsito a los enfoques institucionales. Revista española de pedagogía, 72(257).

Zelaya, V. \& Campanario, J. (2001). Concepciones de los profesores nicaragüenses de física en el nivel de secundaria sobre la ciencia, su enseñanza y su aprendizaje. Revista electrónica interuniversitaria de formación del profesorado, 4(1), 6. 
